\title{
String order and symmetries in quantum spin lattices
}

\author{
D. Pérez-García ${ }^{1}$, M.M. Wolf ${ }^{2}$, M. Sanz ${ }^{2}$, F. Verstraete ${ }^{3}$, J.I. Cirac ${ }^{2}$ \\ 1 Departamento de Analisis Matematico. Universidad Complutense de Madrid, 28040 Madrid, Spain. \\ 2 Max-Planck Institut für Quantenoptik, Hans-Kopfermann-Str. 1, Garching, D-85748, Germany \\ 3 Fakultät für Physik, Universität Wien, Boltzmanngasse 5, A-1090 Wien, Austria.
}

(Dated: October 25, 2018)

\begin{abstract}
We show that the existence of string order in a given quantum state is intimately related to the presence of a local symmetry by proving that both concepts are equivalent within the framework of finitely correlated states. Once this connection is established, we provide a complete characterization of local symmetries in these states. The results allow to understand in a straightforward way many of the properties of string order parameters, like their robustness/fragility under perturbations and their typical disappearance beyond strictly one-dimensional lattices. We propose and discuss an alternative definition, ideally suited for detecting phase transitions, and generalizations to two and more spatial dimensions.
\end{abstract}

PACS numbers: 03.67.-a, 64.70.Tg, 71.10.Hf, 75.10.Pq

Order parameters play a crucial role in describing the different phases of matter. However, there exist some phases which, despite displaying very intriguing features, are not amenable of such a description. In some cases it is nevertheless possible to introduce more sophisticated quantities that are able to characterize those phases. A paradigmatic example is given by the string order parameter (SOP), which reveals the appearance of a hidden order (so-called string order, $\mathrm{SO}$ ) in certain spin systems [1, 2, 3]. This quantity can be expressed as an expectation value of some non-local operator, and the appearance of SO is highlighted by a non-vanishing value in the thermodynamical limit. Despite its importance, we still do not have a systematic characterization of its properties. It is not clear under which conditions SO appears in a $1 \mathrm{D}$ system, for which kind of small perturbations respecting the gap it is robust [4], or when it can be used to detect a quantum phase transition. Apart from that, it seems that the SO looses some of its desirable properties beyond strictly one-dimensional systems $[\underline{3}, 4]$.

In this work we clarify all those questions for finitely correlated states (FCS) [6], i.e., matrix product states (MPS) [5] on infinite chains. The relevance of these states relies on the fact that every quantum state of a finite system has an exact MPS representation [5, 7] and that ground states of $1 \mathrm{D}$ short-range interactions can be efficiently approximated within this class [9]. In this framework we will show that the appearance of $\mathrm{SO}$ is intimately related to the existence of symmetries, which explains how it can be used to detect quantum phase transitions. We propose another parameter which better recognizes this appearance, since it does not have some of the shortcomings of the SOP. We also provide a natural generalization of SO to higher dimensional lattices (membrane order) which retains all the desired properties. Finally, we give several examples displaying a large variety of phenomena.

1D Chains: String order. We will mostly consider infinite chains of identical spin- $S$ particles in a translationally invariant state $\Psi$. We say that the state $\Psi$ has string order if there exist a local unitary $u \neq \mathbb{1}$ and local operators $x, y$ (which can be taken hermitian) such that

$$
\begin{aligned}
\lim _{N \rightarrow \infty}\left|S_{N}(x, y, u, \Psi)\right| & >0, \\
S_{N}(x, y, u, \Psi) & :=\left\langle\Psi\left|x \otimes u^{\otimes N} \otimes y\right| \Psi\right\rangle .
\end{aligned}
$$

Later on we will introduce alternative quantities which will extend this definition [18].

Matrix product states of $L$ spins in a translationally invariant state have the form

$$
\left|\Psi_{L}\right\rangle=\sum_{n_{1} \ldots n_{L}=-S}^{S} \operatorname{tr}\left(A_{n_{1}} \ldots A_{n_{L}}\right)\left|n_{1}, \ldots, n_{n}\right\rangle,
$$

where the $A$ 's are $D \times D$ matrices. We will write $\Psi_{\infty}$ for the thermodynamic limit meaning that $L \rightarrow \infty$ is taken after the expectation value. In this limit the states are known as finitely correlated states (FCS) [ $[$ - - the subject of our studies. Most of the properties of these states are encoded in a linear map defined as

$$
\mathcal{E}(X)=\sum_{n=-S}^{S} A_{n} X A_{n}^{\dagger} .
$$

The matrices $A$ can always be chosen such that

$$
\mathcal{E}(\mathbb{1})=\mathbb{1}, \quad \mathcal{E}^{*}(\Lambda)=\Lambda,
$$

where $\mathcal{E}^{*}$ denotes the map which is obtained by interchanging $A_{n} \leftrightarrow A_{n}^{\dagger}$ in (4), $\Lambda \geq 0$ and $\operatorname{tr}(\Lambda)=1$. Thus, $\mathcal{E}\left(\mathcal{E}^{*}\right)$ is a unital (trace-preserving) completely positive map, i.e., a quantum channel, and has an eigenvalue equal to 1 . A FCS is a pure state iff $\Lambda>0$ and $\mathcal{E}$ has only one eigenvalue of modulus one. Since there is a unique decomposition [6] of mixed FCS into pure ones and a mixed FCS has SO iff one of its pure components has it, we will in the following restrict to pure FCS. Note that all of 
them are unique ground states of gapped finite-range interactions.

String order and finitely correlated states. For any unitary $u$ the SOP (2) of a FCS is most easily expressed by introducing a map

$$
\mathcal{E}_{u}(X):=\sum_{n, n^{\prime}}\left\langle n^{\prime}|u| n\right\rangle A_{n} X A_{n^{\prime}}^{\dagger}=\sum_{j} e^{i \theta_{j}} \tilde{A}_{j} X \tilde{A}_{j}^{\dagger},
$$

where $\tilde{A}_{j}=\sum_{n}\langle\tilde{j} \mid n\rangle A_{n}$, and $u=\sum_{j} e^{i \theta_{j}}|\tilde{j}\rangle\langle\tilde{j}|$. Then [5]

$$
S_{N}\left(x, y, u, \Psi_{\infty}\right)=\operatorname{tr}\left[\Lambda \mathcal{E}_{x} \mathcal{E}_{u}^{N} \mathcal{E}_{y}(\mathbb{1})\right]
$$

where $\mathcal{E}_{x, y}$ are defined analogous to $\mathcal{E}_{u}$ in Eq.(6).

The following Lemma studies the spectral radius $\rho$ of $\mathcal{E}_{u}$ which is crucial for Eq.(7) due to the limit $N \rightarrow \infty$.

Lemma $1 \rho\left(\mathcal{E}_{u}\right) \leq 1$ with equality iff there exists a unitary $V$ and $\theta \in[0,2 \pi)$ such that

$$
V^{\dagger} \tilde{A}_{j}=e^{i\left(\theta-\theta_{j}\right)} \tilde{A}_{j} V^{\dagger}
$$

$\mathcal{E}_{u}$ has at most one eigenvalue of modulus 1.

Proof: Let us consider an eigenvector, $V$, of $\mathcal{E}_{u}$ with eigenvalue $\lambda$, i.e. $\mathcal{E}_{u}(V)=\lambda V$. Multiplying from the right by $\Lambda V^{\dagger}$ and taking traces, we obtain

$$
\begin{aligned}
& |\lambda| \operatorname{tr}\left(V \Lambda V^{\dagger}\right)=\left|\sum_{j} e^{i \theta_{j}} \operatorname{tr}\left(\tilde{A}_{j} V \tilde{A}_{j}^{\dagger} \Lambda V^{\dagger}\right)\right| \\
\leq & {\left[\sum_{j} \operatorname{tr}\left(V \tilde{A}_{j}^{\dagger} \Lambda \tilde{A}_{j} V^{\dagger}\right)\right]^{1 / 2}\left[\sum_{j} \operatorname{tr}\left(\tilde{A}_{j}^{\dagger} V \Lambda V^{\dagger} \tilde{A}_{j}\right)\right]^{1 / 2} } \\
= & \operatorname{tr}\left(V \Lambda V^{\dagger}\right)
\end{aligned}
$$

where we have used Cauchy-Schwarz inequality and (5). Since $\Lambda>0, \operatorname{tr}\left(V \Lambda V^{\dagger}\right)>0$ and thus $|\lambda| \leq 1$ as stated. Now, if condition (8) is fulfilled one can readily see that $e^{i \theta}$ is an eigenvalue of $\mathcal{E}_{u}$ by using Eq. (6), and thus $\rho\left(\mathcal{E}_{u}\right)=1$. On the contrary, if $|\lambda|=1$, then the inequality in (9) has to become an equality, and thus $\alpha e^{i \theta_{j}} \Lambda^{1 / 2} V^{\dagger} \tilde{A}_{j}=\Lambda^{1 / 2} \tilde{A}_{j} V^{\dagger}$. Multiplying by the adjoint expression, taking traces, summing in $j$, and using again (5) one obtains that $|\alpha|=1$, i.e. $\alpha=e^{-i \theta}$. Since $\Lambda$ is invertible we obtain (8). This also implies that

$$
\mathcal{E}\left(V^{\dagger} V\right)=V^{\dagger} \sum_{j} \tilde{A}^{j} \tilde{A}^{j \dagger} V=V^{\dagger} V
$$

where we have used (5). Since $\mathbb{1}$ is the only fixed point of $\mathcal{E}$, we get $V^{\dagger} V=\mathbb{1}$. Moreover, suppose that $\mathcal{E}_{u}$ has two eigenvectors, $V, V^{\prime}$ with eigenvalues $e^{i \theta}, e^{i \theta^{\prime}}$, respectively. Then, using (9) we have

$$
\mathcal{E}\left(V^{\dagger} V^{\prime}\right)=\sum_{j} \tilde{A}_{j} V^{\dagger} V^{\prime} \tilde{A}_{j}^{\dagger}=e^{i\left(\theta^{\prime}-\theta\right)} V^{\dagger} V^{\prime},
$$

such that the same argument gives $V=V^{\prime}$ and $\theta=\theta^{\prime}$ $\square$.

Now we can specify the conditions required for SO. First, $\rho\left(\mathcal{E}_{u}\right)=1$ since otherwise $S_{N}$ will decay exponentially with $N$. Using Lemma 1 we know that the eigenvalue $\lambda$ of magnitude 1 is not degenerate, so let us denote by $V$ and $Y$ the corresponding right and left eigenvectors, i.e. $\mathcal{E}_{u}(V)=\lambda V, \mathcal{E}_{u}^{*}(Y)=\lambda Y$, where $\mathcal{E}_{u}^{*}$ is again given by expression (6) but interchanging $A_{n} \leftrightarrow A_{n}^{\dagger}$. We have $\lim _{N \rightarrow \infty} S_{N}\left(x, y, u, \Psi_{\infty}\right)=\operatorname{tr}\left[Y \mathcal{E}_{y}(\mathbb{1})\right] \operatorname{tr}\left[\Lambda \mathcal{E}_{x}(V)\right]$. Writing $\mathcal{E}_{u}^{*}(Y) V=\lambda Y V$ and using (8) we arrive to the conclusion that $Y=\Lambda V^{\dagger}$. Thus, the conditions for the SOP not to vanish are: (i) $\rho\left(\mathcal{E}_{u}\right)=1$; (ii) $\operatorname{tr}\left[\Lambda V^{\dagger} \mathcal{E}_{y}(\mathbb{1})\right]$, $\operatorname{tr}\left[\Lambda \mathcal{E}_{x}(V)\right] \neq 0$.

We may ask ourselves if the condition $\rho\left(\mathcal{E}_{u}\right)=1$ is sufficient to have SO, i.e. if there are always two operators $x$ and $y$ such that the other conditions are fulfilled. To answer this question we notice that $\operatorname{tr}\left[\Lambda V^{\dagger} \mathcal{E}_{y}(\mathbb{1})\right]^{*}=$ $\operatorname{tr}\left[\Lambda \mathcal{E}_{z}(V)\right]$, where $z=\tilde{u} y$ and $\tilde{u}=\sum_{j} e^{i\left(\theta_{j}-\theta\right)}|\tilde{j}\rangle\langle\tilde{j}|$. Thus, we can always choose $y=x^{\dagger} \tilde{u}$ so that condition (ii) above is simplified to $\operatorname{tr}\left[\Lambda \mathcal{E}_{x}(V)\right] \neq 0$. It is clear that it suffices that $\operatorname{tr}\left(V \Lambda A_{n} A_{m}^{\dagger}\right) \neq 0$ for some $n, m$ since then we can simply choose $x=|n\rangle\langle m|$. We conclude that

Theorem 1 For a pure FCS there exists SO iff there exist a unitary $\tilde{u} \neq \mathbb{1}, V$, and $n, m$ such that

$$
\mathcal{E}_{\tilde{u}}(V)=V, \quad \operatorname{tr}\left(V \Lambda A_{n} A_{m}^{\dagger}\right) \neq 0 .
$$

Now, we show that the second condition can be dropped in two situations. First, if $x$ and $y$ in (2) are products of observables acting on $D^{2}$ spins. The reason is that the set $S_{D}:=\operatorname{span}\left\{A_{n_{1}} \ldots A_{n_{D}} A_{m_{D}}^{\dagger} \ldots A_{m_{1}}^{\dagger}\right\}$ spans the set of $D \times D$ matrices, so that there is always a linear combination $X$ of these matrices for which $\operatorname{tr}(V \Lambda X) \neq 0$. To see that this set is complete note first that $S_{m-1} \subseteq S_{m}$ since $\sum_{n_{D}} A_{n_{1}} \ldots A_{n_{D}} A_{n_{D}}^{\dagger} \ldots A_{m_{1}}^{\dagger}=$ $A_{n_{1}} \ldots A_{n_{D-1}} A_{m_{D .1}}^{\dagger} \ldots A_{m_{1}}^{\dagger}$. This inclusion must be strict unless $m>D^{2}$ since $S_{m-1}=S_{m}$ implies $S_{m}=$ $S_{m+1}$ and for a sufficient large $N$ the set $\left\{A_{n_{1}} \ldots A_{n_{N}}\right\}$ must span the entire space of matrices [5]. Another situation is the one in which there exists a continuous group of unitaries $V$ fulfilling the first condition, i.e., we can parametrize $V=e^{i \phi H}$. Then, we can always choose $\phi$ sufficiently small such that $\operatorname{tr}(V \Lambda) \neq 0$ and this suffices since we have (5).

Symmetries in finitely correlated states. We say that a state has a local symmetry if there is a unitary $u \neq \mathbb{1}$ such that

$$
u \otimes \cdots \otimes u|\Psi\rangle=|\Psi\rangle
$$

This formally means that for every $N$-site reduced density operator $\varrho$ we have $u^{\otimes N} \varrho u^{\dagger \otimes N}=\varrho$.

For FCS the condition $\rho\left(\mathcal{E}_{u}\right)=1$ is not only equivalent to having $\mathrm{SO}$ - as we saw in the previous section - it is also equivalent to the presence of a local symmetry: 
Theorem 2 A pure FCS has a local symmetry $u$ iff $\rho\left(\mathcal{E}_{u}\right)=1$.

Proof: If $\rho\left(\mathcal{E}_{u}\right)=1$ the result is a direct consequence of Lemma 1 which implies that $V^{\dagger} \Lambda V=\Lambda$. The converse follows from the fact that

$$
\frac{1}{D^{2}} \leq \operatorname{tr}\left(\varrho^{2}\right)=\operatorname{tr}\left[\varrho u^{\otimes N} \varrho u^{\dagger \otimes N}\right]=\operatorname{tr}\left[L\left(\mathcal{E}_{u} \otimes \mathcal{E}_{u^{\dagger}}\right)^{N}(R)\right],
$$

for some $L, R$ which are independent of $N$.

This theorem, together with Lemma 1 provides a complete characterization of FCS with local symmetries. We note that an analogous statement can be found (though without proof) in Ref. []. .

Equivalent criteria for the existence of a local symmetry can be given in terms of the isometry $B:=\sum_{j}|j\rangle A_{j}$ as well as for the $D^{2} \times D^{2}$ matrix $E:=\sum_{j} A_{j} \otimes \bar{A}_{j}$. In all cases $V$ and $u$ are elements of two unitary representations of a symmetry group (different from the identity):

- Condition $\mathrm{C} 1:(u \otimes \mathbb{1}) B=(\mathbb{1} \otimes V) B V^{\dagger}$.

- Condition $\mathrm{C} 2: \mathcal{E}$ is covariant, i.e., for all $X$, $\mathcal{E}\left(V X V^{\dagger}\right)=V \mathcal{E}(X) V^{\dagger}$.

- Condition C3: $[E,(V \otimes \bar{V})]=0$.

C1 can be obtained from Lemma 1 by using the spectral decomposition of $u$. $\mathrm{C} 2$ and $\mathrm{C} 3$ are the same if we use $\langle k, l|E| i, j\rangle=\langle k|\mathcal{E}(|i\rangle\langle j|)| l\rangle$. It is also clear that Lemma 1 implies C2. Finally, if C3 is fulfilled, then we have that $V A_{j} V^{\dagger}$ are also Kraus operators of the map $\mathcal{E}$. Since all Kraus decompositions are related by a unitary matrix, say $u$, we have that $V A_{j} V^{\dagger}=\sum_{n}\langle n|u| j\rangle A_{n}$ implying the condition of Lemma 1.

Now, we can use C3 to derive a criterion for the existence of a continuous symmetry where $V=e^{i \phi H}$. Expanding in first order in $\phi$ we obtain

$$
M(H):=[E, H \otimes \mathbb{1}-\mathbb{1} \otimes \bar{H}]=0 .
$$

Since $M$ is a linear map on the space of hermitian matrices, we have that: a pure FCS has a local continuous symmetry iff $M$ has a non-trivial kernel.

Similar criteria can be given for discrete symmetries, e.g., $\mathbb{Z}_{2}$ symmetry where the $A$ 's are either block diagonal or block off-diagonal [14].

Alternative definitions. One can understand the importance of the SOP to detect quantum phase transition in terms of its relation to local symmetries. If we have a Hamiltonian with certain local symmetry and its ground state is unique, then there may be SO. If we change the parameters of the Hamiltonian but keeping the symmetry until the gap closes, then the ground state will be degenerate and the symmetry may be broken [19]. Thus, the SO may disappear at that point, indicating the presence of the transition. Note that due to the possible choices of the operators $x$ and $y$ in the definition (2), it may happen that the SOP for a particular choice vanishes even if there still is a symmetry. In order to avoid this, one may look at the quantity

$$
R_{L}(u):=\left\langle\Psi_{L}\left|u^{\otimes L}\right| \Psi_{L}\right\rangle,
$$

where $\left|\Psi_{L}\right\rangle$ is the ground state of the Hamiltonian acting on $L$ sites with periodic boundary conditions (Eq. (3) in the case of MPS), which is indeed directly related to the existence of a symmetry. In fact, $R_{\infty}(u)=\lim _{L} R_{L}(u)$ can only vanish if the gap is closed, and thus it is ideally suited to study the presence of transitions. Note also that it can be straightforwardly determined from numerical algorithms based on MPS [10].

Example 1: AKLT state. It is instructive to revise the appearance of $\mathrm{SO}$ in the ground state of the AKLT model [11]. For that state we have $S=1, A^{0}=\sigma_{z} / \sqrt{3}$ and $A^{1,-1}=\sqrt{2 / 3} \sigma_{ \pm}$where the sigmas denote Pauli matrices. One finds $\Lambda=\mathbb{1}$, and taking $u=e^{i \pi S_{z}}$ we obtain $V=\sigma_{z}$ and we can take $x=y=S_{z}$, so that $S_{L}\left(x, y, u, \Psi_{\infty}\right)=-4 / 9$ [1]. Note that the AKLT state has $S U(2)$ symmetry so that obviously $R_{L}(u)=1$.

Example 2: Cluster state. We have [5]

$$
A^{0}=\frac{1}{\sqrt{2}}\left(\begin{array}{ll}
1 & 1 \\
0 & 0
\end{array}\right) \quad A^{1}=\frac{1}{\sqrt{2}}\left(\begin{array}{cc}
0 & 0 \\
1 & -1
\end{array}\right) .
$$

This has the symmetry induced by $u=-\sigma_{x}$. We have $V=\sigma_{y}$, and one can readily see that $\operatorname{tr}\left(V \Lambda A_{n} A_{j}^{\dagger}\right)=0$. Thus, there is no SO. However, if we take two particles, we can choose $x=\sigma_{z} \otimes \sigma_{y}$ and $y=\sigma_{y} \otimes \sigma_{z}$, so that the SOP is one. In general, we have $R_{L}(u)=1$ as expected.

2D Systems: Membrane order. Since the existence of a local symmetry, and the possibility of determining it numerically via the quantity $R_{L}$ defined in (14), seems to be an appropriate definition of SO in 1D systems, we will now try to extend it to $2 \mathrm{D}$. In this case there is more freedom in the choice of locations for the local unitaries $u$. First we can let them act on the whole lattice. If this leaves the state invariant (or the respective $R_{L}$ is not vanishing) we will say that we have membrane order (MO). We can also put them as a string of operators, in which case we will talk about SO [20], or even in more sophisticated configurations, as a band of operators (BO). Note that the MO so defined shares all the desired properties for the $\mathrm{SO}$ in $1 \mathrm{D}$ and thus provides a natural generalization to higher dimensions. In particular, it should not exhibit spontaneous breakdown when switching on couplings in the second dimension as pointed out in [4] for the SO.

To gain more inside we will consider the generalization of MPS/FCS called projected entangled pair states (PEPS) 12, 13] where the matrices $A_{k}$ are replaced by tensors $B_{k}$ whose degree depends on the geometry of the lattice. For these states local symmetries can arise in a 
a)

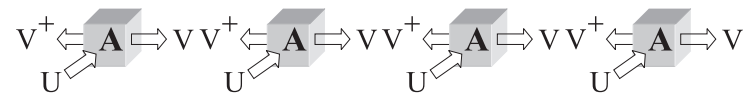

b)
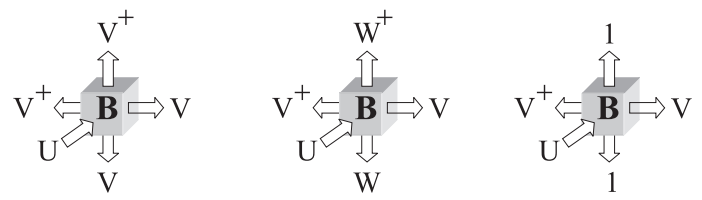

FIG. 1: (a) Symmetries in FCS/MPS. The tensor $A$ has three indices, one corresponding to the physical spin (pointing in) and two for the virtual systems (pointing left and right). Applying $U$ to the physical index is equivalent to applying $V$ to the virtual ones. Since the tensor are contracted in a line the unitaries $V$ and $V^{\dagger}$ cancel and thus the state does not change. (b) $2 \mathrm{D}$ generalization (square lattice). The tensor $B$ has one physical and four virtual indices. When applying $U$ to the former, we can have different effects on the virtual system which will, after contraction, leave the state invariant. The example on the right allows for SO.

similar way as in the 1D case (Fig 1). We interpret each tensor as an operator $B=\sum_{s}|s\rangle\left\langle\phi_{s}\right|:\left(H_{D}\right)^{\otimes 4} \rightarrow H_{S}$, where $H_{S}\left(H_{D}\right)$ is the Hilbert space corresponding to the physical (virtual) spins. The PEPS exhibits a local symmetry if there exist unitaries $V_{k}$ and $U \neq \mathbb{1}$ with

$$
U B=B V_{1} \otimes \ldots \otimes V_{4},
$$

such that when contracting the indices of $B$ to create the state $\Psi$ (analogous to the matrices $A$ in Eq.(3)) the $V$ 's cancel [see Fig. 1]. Thus, we will have $\left\langle\Psi\left|U^{\otimes N}\right| \Psi\right\rangle=|\Psi\rangle$ and therefore MO. Note that we have the possibility of having different $V$ 's, in contrast to what happens in 1D. This structure also allows us to understand the (dis-) appearance of $\mathrm{SO}$ or $\mathrm{BO}$. However, in the $2 \mathrm{D}$ case, the connection between the existence of a local symmetry and Eq. (16) is less straight and will be analyzed in detail elsewhere [14].

Example 3: AKLT state [1]. In this case $S=2$, $\left|\phi_{s}\right\rangle=\left(\sigma_{y} \otimes \sigma_{y} \otimes 1 \otimes 1\right)\left|\bar{\phi}_{s}\right\rangle$ and $\phi_{s}$ is an orthonormal basis of the symmetric subspace of $\left(\mathbb{C}^{2}\right)^{\otimes 4}$. $U$ and $V$ correspond to a 5 and 2-dimensional representation of $S U(2)$, and thus fulfill the condition of MO. One can prove that there is no $\mathrm{SO}$ even for a simple ladder formed by two chains.

Example 4: Cluster state [15]. Here, $S=1 / 2$ and $\left|\phi_{-1 / 2}\right\rangle=|++00\rangle,\left|\phi_{1 / 2}\right\rangle=|--11\rangle$ with $| \pm\rangle=|0\rangle \pm|1\rangle$. One can take $U=\sigma_{x}, V_{u}=V_{r}=\sigma_{x}$ and $V_{d}=V_{l}=\sigma_{z}$. But one can also take $U=\sigma_{z}$, three of the $V$ 's equal to $\mathbb{1}$ and decide either $V_{u}=\sigma_{z}, V_{r}=\sigma_{z}, V_{d}=\sigma_{x}$ or $V_{l}=\sigma_{x}$. As a consequence, there is MO. One can readily show that there is no $\mathrm{SO}$. However still there is a $\mathrm{BO}$, in the sense that this state is an eigenstate of the operator obtained by applying unitaries to three consecutive lines ( $\sigma_{z}$ to the first and the third and $\sigma_{x}$ to the middel one).
Example 5: Toric code [16]. This is a state with $S=1 / 2$ and we have alternative tensors in the A and $B$ sublattices, In this case we have alternating projectors $B=|0\rangle\left\langle\left.\Phi_{+} \Phi_{+}\right|_{u l d r}+\mid 1\right\rangle\left\langle\left.\Phi_{-} \Phi_{-}\right|_{u l d r}\right.$ and $B^{\prime}=$ $|0\rangle\left\langle\left.\Phi_{+} \Phi_{+}\right|_{\text {urld }}+\mid 1\right\rangle\left\langle\left.\Phi_{-} \Phi_{-}\right|_{\text {urld }}\right.$, resp. One can take $U=\sigma_{x}, V_{l}=V_{r}=\sigma_{z}$ and $V_{u}=V_{d}=\mathbb{1}$. Since there is no unitary to be cancelled in the up and down positions, it has SO (and also MO).

Conclusion. We have shown that the existence of string order is, in the framework of FCS, equivalent to the existence of a local symmetry. This gives a direct explanation of many of its intriguing features, like its robustness/fragility under perturbations or the capability of detecting phase transitions. Though the FCS case provides evidence for the generality of this equivalence it remains an open problem to extend this beyond FCS, e.g., to all ground states of gapped local Hamiltonains. We have characterized the existence of local symmetries in FCS, related it to intertwining isometries and covariant channels ( $\mathrm{C} 1$ and $\mathrm{C} 2$ ), and sketched the case of $2 \mathrm{D}$ systems through PEPS. The list of examples can easily be extended and shows, for instance, in a simple way the presence of string order in topological ordered states, as it is illustrated in Kitaev's toric code.

The present work is an example that MPS/FCS and PEPS are not only useful for numerical algorithms, but also to prove and clarify interesting statements.

The obtained results shed light on the role of symmetries in spin systems also in other contexts like Lieb-Schultz-Mattis type theorems [17]. The respective relation between integer/half-integer spins and irreducible/reducible representations will be discussed in a forthcoming paper [14] together with a more detailed investigation of the conditions for symmetry.

The authors thank A.Rosch and E.Altman for discussions. Portions of this work were done at the Workshop on Tensor network methods and entanglement at the Erwin Schrödinger Institute. This work has been supported by the EU project SCALA, the DFG (FOR 635, MAP and NIM) and the Spanish grant MTM2005-00082.

[1] M. den Nijs and K. Rommelse, Phys. Rev. B 40, 4709 (1989).

[2] E. G. Dalla Torre, E. Berg, E. Altman, Phys. Rev. Lett. 97, 260401 (2006); F. Anfuso, A. Rosch Phys. Rev. B 75, 144420 (2007).

[3] Eugene H. Kim, G. Fath, J. Solyom, D. J. Scalapino Phys. Rev. B 62, 14965 (2000); S. Todo, M. Matsumoto, C. Yasuda, H. Takayama, Phys. Rev. B 64, 224412 (2001).

[4] F. Anfuso, A. Rosch Phys. Rev. B 76, 085124 (2007). 
[5] D. Pérez-García, F. Verstraete, M.M. Wolf, J.I. Cirac, Quant. Inf. Comp. 7, 401 (2007).

[6] M. Fannes, B. Nachtergaele and R. F. Werner, Commun. Math. Phys. 144, 443-490 (1992).

[7] G. Vidal, Phys. Rev. Lett. 91, 147902 (2003).

[8] F. Verstraete, M.A. Martin-Delgado, J.I. Cirac, Phys. Rev. Lett. 92, 087201 (2004).

[9] F. Verstraete, J.I. Cirac, Phys. Rev. B 73, 094423 (2006); M. B. Hastings J. Stat. Mech. (2007) P08024.

[10] F. Verstraete, D. Porras, J.I. Cirac, Phys. Rev. Lett 93, 227205 (2004).

[11] I. Affleck, T. Kennedy, E. H. Lieb and H. Tasaki, Commun. Math. Phys. 115, 477 (1988).

[12] F. Verstraete, J.I. Cirac, arXiv:cond-mat/0407066

[13] D. Pérez-García, F. Verstraete, M.M. Wolf, J.I. Cirac, arXiv:0707.2260

[14] In preparation.

[15] R. Raussendorf, H.J. Briegel, Phys. Rev. Lett. 86, 5188 (2001); F. Verstraete, J.I. Cirac, Phys. Rev. A 70,
$060302(\mathrm{R})$ (2004).

[16] A. Yu. Kitaev, Annals Phys. 303, 2 (2003).

[17] E. Lieb, T. Schultz, and D. Mattis, Ann. Phys. 16 (1961), 407466; M. B. Hastings, Phys.Rev. B 69 (2004) 104431; B. Nachtergaele, R. Sims, Commun. Math. Phys. 276 (2007) 437-472.

[18] In the context of MPS one finds an alternative but equivalent definition for string order [8] involving a finite chain with access to the virtual levels in the endpoints.

[19] If one perturbs the Hamiltonian without keeping the symmetry, it is clear from our picture that the string order will vanish, even if the gap does not close. This explains the fragility of the SO (cf. [4] )

[20] In this case one can obtain again the equivalence with the 'classical' definition of string order. In order to see this, it is enough to invoke the injectivity condition defined in [13]. 